\title{
Algebraic Lens Distortion Model Estimation
}

\author{
Luis Alvarez ${ }^{1}$, Luis Gomez ${ }^{2}$, Rafael Sendra ${ }^{3}$ \\ ${ }^{1}$ Dep. Informática y Sistemas, Universidad de Las Palmas de Gran Canaria, Spain (lalvarez@ulpgc.es) \\ 2 Dep. Ingeniería Electrónica y Automática, Universidad de Las Palmas de Gran Canaria, Spain (lgomez@ctim.es) \\ ${ }^{3}$ Dep. Matemáticas, Universidad de Alcalá, Spain (rafael.sendra@uah.es) \\ Communicated by Pascal Monasse Demo edited by Nicolas Limare
}

\begin{abstract}
A very important property of the usual pinhole model for camera projection is that $3 \mathrm{D}$ lines in the scene are projected to 2D lines. Unfortunately, wide-angle lenses (specially low-cost lenses) may introduce a strong barrel distortion, which makes the usual pinhole model fail. Lens distortion models try to correct such distortion. We propose an algebraic approach to the estimation of the lens distortion parameters based on the rectification of lines in the image. Using the proposed method, the lens distortion parameters are obtained by minimizing a 4 total-degree polynomial in several variables. We perform numerical experiments using calibration patterns and real scenes to show the performance of the proposed method.
\end{abstract}

\section{Source Code}

The source code, the code documentation, and the online demo are accessible at the IPOL web page of this article ${ }^{1}$. In this page, an implementation is available for download. Compilation and usage instructions are included in the README.txt file of the archive.

Keywords: lens distortion; wide-angle lens; Hough transform; line detection.

\section{Introduction}

The basic standard model for barrel and pincushion distortion compensation is a radial distortion model given by the following expression

$$
\left(\begin{array}{c}
\hat{x}-x_{c} \\
\hat{y}-y_{c}
\end{array}\right)=L(r)\left(\begin{array}{c}
x-x_{c} \\
y-y_{c}
\end{array}\right),
$$

where $(x, y)$ are the original point coordinates (distorted), $(\hat{x}, \hat{y})$ are the corrected (undistorted) point coordinates, $\left(x_{c}, y_{c}\right)$ is the center of the camera distortion model, usually located at the image center. The distance from any image point to the center of the camera distortion model is given by

\footnotetext{
${ }^{1}$ https://doi.org/10.5201/ipol.2010.ags-alde
} 


$$
r=\sqrt{\left(x-x_{c}\right)^{2}+\left(y-y_{c}\right)^{2}},
$$

and $L(r)$ is the function defining the shape of the distortion model, which is usually approximated by a Taylor expansion as

$$
L(r)=k_{0}+k_{1} r+k_{2} r^{2}+k_{3} r^{3}+\cdots,
$$

where the set

$$
\mathbf{k}=\left(k_{0}, k_{1}, \ldots, k_{N_{k}}\right)^{T},
$$

gathers the distortion parameters. The complexity of the model is given by the number of terms of the Taylor expansion we use to approximate $L(r)$.

We use the general approach to determine $\mathrm{L}(\mathrm{r})$ by imposing the requirement that the projection of 3D lines has to be 2D straight lines in the image. This approach has been successfully used in previous works [1] to minimize the objective error functions which are expressed in terms of line equations or distance functions.

In this paper we propose a new fast technique to obtain the distortion parameter model using a new lens distortion measure error. The main advantage of our formulation is that it yields a general 4-degree polynomial in the distortion parameters $\mathbf{k}$ that can be minimized using powerful techniques of computer algebra in one step for the 3 parameter case

$$
L(r)=k_{0}+k_{1} r+k_{2} r^{2},
$$

which is, in most cases, sufficient for image distortion correction.

The lens distortion is included in the camera calibration model in the following manner: given a camera defined by a rotation matrix $\mathrm{R}$, a focus c, and a $3 \times 3$ intrinsic matrix parameter $\mathrm{A}$, the projection of a $3 \mathrm{D}$ point $\mathrm{X}$ in the camera is given by the following expression

$$
\left.\left(\begin{array}{c}
\hat{x} \\
\hat{y} \\
1
\end{array}\right)=a A(R,-R c)\right)\left(\begin{array}{c}
X \\
Y \\
Z \\
1
\end{array}\right),
$$

where $\mathrm{s}$ is the usual projective factor value. In the case of $L(r) \equiv 1$, the camera model is lens distortion free and the above expression becomes the usual "pinhole" projective model.

We observe that lens distortion correction is performed in pixel image coordinates. In order for the lens distortion model to be a radial function in pixels coordinates we need to assume that the camera CCD sensor has square pixels. This square pixels assumption is satisfied, in practice, for most modern digital cameras. On the other hand, this assumption could be removed if we use normalized coordinates instead of pixel coordinates, but to normalize the point coordinates we need to know the camera intrinsic parameters. Since we assume that camera intrinsic parameters are unknowns we have formulated the lens distortion correction in terms of pixel coordinates.

\section{A New Energy Function to Measure the Distortion Error}

Previous works correct the distortion by minimizing the RMS distance for all the 2D projected points to the a priori known $N$ lines. This minimization can be carried out through any optimization method (gradient-like). For these cases, the energy function is as follows

$$
D(\mathbf{k})=\frac{1}{N} \sum_{l=1}^{N} \frac{1}{N_{l}} \sum_{i=1}^{N_{l}} \frac{\left(a_{l} \hat{x}_{l, i}+b_{l} \hat{y}_{l . i}+c_{l}\right)^{2}}{a_{l}^{2}+b_{l}^{2}}
$$


where $\left(\hat{x}_{l, i}, \hat{y}_{l, i}\right)$, are the undistorted points using the distortion model provided by $\mathbf{k}$, and where $a_{l} \hat{x}_{l, i}+b_{l} \hat{y}_{l . i}+c_{l}=0$ is the line that minimizes $D(\mathbf{k})$ for a given choice of points $\left(\hat{x}_{l, i}, \hat{y}_{l, i}\right)_{i=1, . ., N_{l}}$.

This energy function is not necessarily convex and any optimizer will have to deal with an unknown number of local minimizers, which, as a result, will provide a local solution, therefore, not the optimal. Since this energy function is widely applied, we have added it to our code for comparison.

Our proposal is quite different, and, as will be demonstrated by the results, more efficient. We define an energy function to measure the distortion error built on statistical measure. Given the set of points, we calculate the covariance matrix $\mathrm{S}$ as

$$
\hat{S}^{l}(\mathbf{k})=\left(\begin{array}{cc}
\hat{S}_{x x}^{l} & \hat{S}_{x y}^{l} \\
\hat{S}_{x y}^{l} & \hat{S}_{y y}^{l}
\end{array}\right)=\frac{1}{N_{l}}\left(\begin{array}{cc}
\sum_{i=1}^{N_{l}}\left(\hat{x}_{l, i}-\overline{\hat{x}_{l, i}}\right)^{2} & \sum_{i=1}^{N_{l}}\left(\hat{x}_{l, i}-\overline{\hat{x}_{l, i}}\right)\left(\hat{y}_{l, i}-\overline{\hat{y}_{l, i}}\right) \\
\sum_{i=1}^{N_{l}}\left(\hat{x}_{l, i}-\overline{\hat{x}_{l, i}}\right)\left(\hat{y}_{l, i}-\overline{\hat{y}_{l, i}}\right) & \sum_{i=1}^{N_{l}}\left(\hat{y}_{l, i}-\overline{\hat{y}_{l, i}}\right)^{2}
\end{array}\right) .
$$

From that, we propose a new distortion measure error as

$$
\hat{E}(\mathbf{k})=\frac{1}{N} \sum_{l=1}^{N} \hat{S}_{x x}^{l} \hat{S}_{y y}^{l}-\left(\hat{S}_{x y}^{l}\right)^{2}
$$

It can be easily proven (see [1]), that this new energy function is always positive and equals 0 if and only if for each line its points are aligned. This is the functional to be minimized to correct the image distortion and the variables are the distortion parameters $\mathbf{k}$.

Besides, if we expand this functional to account for every line and every point, it can be expressed as a 4-degree homogeneous polynomial in the variable $\mathbf{k}$

$$
\hat{E}(\mathbf{k})=\frac{1}{N} \sum_{l=1}^{N} \mathbf{k}^{T} A^{l} \mathbf{k} \mathbf{k}^{\mathbf{T}} B^{l} \mathbf{k}-\mathbf{k}^{T} C^{l} \mathbf{k} \mathbf{k}^{T} C^{l} \mathbf{k},
$$

with

$$
\begin{aligned}
& A_{m, n}^{l}=\frac{1}{N_{l}} \sum_{i=1}^{N_{l}}\left(\left(r_{l, i}\right)^{m} x_{l, i}-\overline{\left(r_{l, i}\right)^{m} x_{l, i}}\right)\left(\left(r_{l, i}\right)^{n} x_{l, i}-\overline{\left(r_{l, i}\right)^{n} x_{l, i}}\right), \\
& B_{m, n}^{l}=\frac{1}{N_{l}} \sum_{i=1}^{N_{l}}\left(\left(r_{l, i}\right)^{m} y_{i}-\overline{\left(r_{i}\right)^{m} y_{i}}\right)\left(\left(r_{i}\right)^{n} y_{i}-\overline{\left(r_{i}\right)^{n} y_{i}}\right), \\
& C_{m, n}^{l}=\frac{1}{N_{l}} \sum_{i=1}^{N_{l}}\left(\left(r_{l, i}\right)^{m} x_{l, i}-\overline{\left(r_{l, i}\right)^{m} x_{l, i}}\right)\left(\left(r_{l, i}\right)^{n} y_{l, i}-\overline{\left(r_{l, i}\right)^{n} y_{l, i}}\right) .
\end{aligned}
$$

Obviously, the global minimum of the new functional corresponds to the trivial solution $\mathbf{k}=$ 0. To avoid this problem, usually the first distortion parameter is set to one. As it is explained in the implementation section of this paper we use another approach: we fit the first distortion parameter using a zoom factor by minimizing the sum of the square distance between the distorted and undistorted points.

\section{An Efficient Technique to Minimize the Proposed Energy Function}

A detailed algebraic analysis of the new functional is described in [1], and, in this section, we briefly present the algebraic technique we apply to minimize the measure of the distortion error. For 
simplicity in the exposition, we present the results for polynomials with real coefficients, but it must be said that they are valid over more general polynomials rings.

As mentioned above, one needs to minimize the distortion error measure function which is a real polynomial in the variable $\mathbf{k}$. Minimizing a polynomial in several variables can be reduced to compute the solutions of an algebraic system of equations, namely the one generated by its gradient. In our case,

$$
\mathcal{S}:=\left\{\frac{\partial \hat{E}(\mathbf{k})}{\partial k_{i}}=0\right\}_{i=0, \ldots, N_{k}} .
$$

When the polynomial is univariate, say

$$
a_{0}+a_{1} k_{p}+\ldots
$$

one just has to approximate the real roots of the univariate polynomial

$$
\frac{\partial \hat{E}\left(k_{p}\right)}{\partial k_{p}}
$$

However, when more than one variable appears, the problem is not trivial. In order to approach this new situation, one can apply computer algebra techniques to prepare symbolically the algebraic system $\mathrm{S}$ before numerical methods are executed. The two-variable case can be treated by means of symbolic linear algebra techniques while the case of more than two variables requires, in general, abstract algebra techniques. In both cases, the underlying theory comes from algebraic geometry and commutative algebra. To be more precise, we first describe in detail how to approach the problem when two variables are considered, and afterward we give a brief description of how to proceed in the general case.

Let us assume that we are dealing with two variables (note that this is the case when working with two distortion parameters), and that the system $\mathrm{S}$ turns out to be

$$
\mathcal{S}:=\left\{\frac{\partial \hat{E}\left(k_{p}, k_{q}\right)}{\partial k_{p}}=0, \frac{\partial \hat{E}\left(k_{p}, k_{q}\right)}{\partial k_{q}}=0\right\}
$$

To compute the solutions of $S$ we apply the so called resultant-based method. Let us shortly describe this algebraic method.

For this purpose, let $G_{1}\left(k_{p}, k_{q}\right)$ and $G_{2}\left(k_{p}, k_{q}\right)$ be two bivariate polynomials with real coefficients. Choosing one variable, say $k_{q}$, as a main variable, we can write $G_{1}$ and $G_{2}$ as

$$
\begin{aligned}
& G_{1}\left(k_{p}, k_{q}\right)=a_{n}\left(k_{p}\right) k_{q}^{n}+\cdots+a_{1}\left(k_{p}\right) k_{q}+a_{0}\left(k_{p}\right), \\
& G_{2}\left(k_{p}, k_{q}\right)=b_{m}\left(k_{p}\right) k_{q}^{m}+\cdots+b_{1}\left(k_{p}\right) k_{q}+b_{0}\left(k_{p}\right),
\end{aligned}
$$

where $a_{i}\left(k_{p}\right)$ and $b_{i}\left(k_{p}\right)$ are univariate polynomials with real coefficients, and $a_{n}\left(k_{p}\right), b_{m}\left(k_{p}\right)$ are not identically zero, with $n>0$ and $m>0$.

In this situation, the resultant of $G_{1}$ and $G_{2}$ with respect to the variable $k_{q}$ (we denote it 
by $\left.\operatorname{Res}_{k_{q}}\left(G_{1}, G_{2}\right)\right)$ is defined as the determinant of the $(n+m) \times(n+m)$ matrix

$$
\left(\begin{array}{ccccccc}
a_{n}\left(k_{p}\right) & a_{n-1}\left(k_{p}\right) & \ldots & a_{0}\left(k_{p}\right) & 0 & \cdots & 0 \\
0 & a_{n}\left(k_{p}\right) & a_{n-1}\left(k_{p}\right) & \cdots & a_{0}\left(k_{p}\right) & \cdots & 0 \\
\vdots & & \ddots & & & \ddots & \vdots \\
0 & 0 & \cdots & a_{n}\left(k_{p}\right) & a_{n-1}\left(k_{p}\right) & \cdots & a_{0}\left(k_{p}\right)
\end{array}\right\} m
$$

Note that the above resultant is a real univariate polynomial in the variable $k_{p}$. Therefore, the variable $k_{q}$ has been eliminated. This is the situation when dealing with two distortion parameters, which makes this algebraic technique very appealing.

Summarizing, one can derive the following algorithm to compute the real solutions of

$$
\mathcal{S}:=\left\{\frac{\partial \hat{E}\left(k_{p}, k_{q}\right)}{\partial k_{p}}=0, \frac{\partial \hat{E}\left(k_{p}, k_{q}\right)}{\partial k_{q}}=0\right\} .
$$

1. Determine

$$
G_{1}:=\frac{\partial \hat{E}\left(k_{p}, k_{q}\right)}{\partial k_{p}}, G_{2}:=\frac{\partial \hat{E}\left(k_{p}, k_{q}\right)}{\partial k_{q}} .
$$

2. Determine

$$
G\left(k_{p}\right):=\operatorname{Res}_{k_{q}}\left(G_{1}, G_{2}\right)
$$

and approximate the real roots of $G\left(k_{p}\right)$. Let $R=\alpha_{1}, \ldots, \alpha_{s}$ be the set of real roots of $\mathrm{G}$.

3. For each $\alpha \in R$, approximate the common real roots of the univariate polynomials, $G_{1}\left(\alpha, k_{q}\right)$ and $G_{2}\left(\alpha, k_{q}\right)$. Let $R_{\alpha}$ be the set of these real common roots.

4. The real solutions of $S$ are $\left\{\left(\alpha, \beta_{\alpha}: \alpha \in R\right.\right.$ and $\left.\beta_{\alpha} \in R_{\alpha}\right\}$.

In the general case, i.e. when working with $s>2$ variables, say $k_{p_{1}}, \ldots, k_{p_{s}}$ the problem cannot be approached so directly by means of resultants. Nevertheless, one can apply Gröbner basis techniques or multivariate-resultants (refer to [2] for further information). Certainly, Gröbner basis techniques can also be applied to the case of two variables but, in that case, we find more suitable the resultantbased method. The basic idea of Gröbner basis, as a tool for solving algebraic systems, is to provide a new algebraic system of equations equivalent to $S$ (i.e. with the same solutions) but much simpler, and such that it has a suitable structure ("triangular") to compute the solutions. Roughly speaking, Gröbner basis can be seen as a generalization of the Gaussian elimination when the equations are not linear.

\section{Online Demo}

An on-line demo of the algorithm is available at the IPOL web page of this article ${ }^{2}$. The demo allows:

\footnotetext{
${ }^{2}$ https://doi.org/10.5201/ipol.2010.ags-alde
} 
- uploading any color image and correcting the radial distortion by means of the proposed algebraic method,

- obtaining the set of distortion parameters and the value of the minimized energy functions associated to the method.

\section{$5 \quad$ Algorithm}

\subsection{Zoom Factor Estimation}

The distortion parameters $\mathbf{k}$ are computed by initially setting $k_{0}=1$. In order to yield undistorted points as close as possible to the distorted ones we estimate a zoom factor to minimize the sum of the square distance between the distorted and the corrected (undistorted) points. Let $\mathrm{s}$ be such zoom factor, then we have

$$
\left(\begin{array}{c}
\hat{x}-x_{c} \\
\hat{y}-y_{c}
\end{array}\right)=s L(r)\left(\begin{array}{c}
x-x_{c} \\
y-y_{c}
\end{array}\right)
$$

We minimize

$$
H(s)=\sum_{l=1}^{N} \sum_{i=1}^{N_{l}}\left(\hat{x}_{l, i}-x_{l, i}\right)^{2}+\left(\hat{y}_{l, i}-y_{l, i}\right)^{2} .
$$

A straightforward computation leads to

$$
H(s)=\sum_{l=1}^{N} \sum_{i=1}^{N_{l}}\left(s \sum_{j=0}^{N_{k}} k_{j}^{n}\left(r_{l, i}\right)^{j+1}-r_{l, i}\right)^{2} .
$$

The minimum of the above function is attained for

$$
s_{\min }=\frac{\sum_{l=1}^{N} \sum_{i=1}^{N_{l}} \sum_{j=0}^{N_{k}} k_{j}^{n}\left(r_{l, i}\right)^{j+2}}{\sum_{l=1}^{N} \sum_{i=1}^{N_{l}}\left(\sum_{j=0}^{N_{k}} k_{j}^{n}\left(r_{l, i}\right)^{j+1}\right)^{2}} .
$$

And finally we update the polynom $L(r)$ (i.e. k) by multiplying all the coeficients by $s_{\min }$ (that is $\left.k_{j}^{n}=s k_{j}^{n} \forall j\right)$.

An interesting advantage of this approach is that the resolution of the undistorted image is similar to the resolution of the original (distorted) image. This is a very useful property if we need to generate the undistorted image from the original distorted one.

\subsection{Algorithm Description}

The derived algorithm for performing the numerical experiments can be structured in the following steps :

1. Compute the edges of the image using an edge detection algorithm with subpixel precision.

2. Select some collections of edge points corresponding to different 3D straight segments that will be used to fit the distortion parameters.

3. Initialize $\mathbf{k}=(1,0, \ldots, 0)^{T}$. 
4. Choose any pair $p, q \in Z\left(1 \leq p, q, \leq N_{k}\right)$ and optimize $k_{p}, k_{q}$ using the proposed algebraic technique.

5. Update $\mathbf{k}$ using a zoom factor in order for distorted and undistorted points to be as close as possible.

Note that in the demo, the first step is not done in an automatic manner, hence, the user must select directly from the image the points supposed to be aligned.

We observe that steps 4-5 could be repeated in order to iteratively estimate all the coefficients of k.

\subsection{Point Coordinates Normalization}

It is well known that when we deal with algebraic methods it is usually better to normalize the point coordinates before computing the algebraic solution of the problem. Following this strategy, at a fist step, we normalize the edge points $\left(x_{i}, y_{i}\right)$ using the transformation

$$
x_{l, i}^{\prime}=\frac{\left(x_{l, i}-x_{c}\right)}{A} \quad y_{l, i}^{\prime}=\frac{\left(y_{l, i}-y_{c}\right)}{A},
$$

where $A$ is given by

$$
A=\sqrt{\frac{\sum_{l=1}^{N} \sum_{i=1}^{N_{l}}\left(x_{l, i}-x_{c}\right)^{2}+\left(y_{l, i}-y_{c}\right)^{2}}{2\left(N_{1}+N_{2}+\ldots+N_{N}\right)}} .
$$

and we compute the distortion parameters $k_{i}^{\prime}$ for the normalized edge points $\left\{\left(x_{i}^{\prime}, y_{i}^{\prime}\right)\right\}_{i=1}^{N}$.

Finally, in order to recover the distortion parameters $k_{i}$ for the original edge points we have just to take into account that following the above expressions and (1) we have that

$$
k_{j}=\frac{k_{j}^{\prime}}{(A)^{j}} .
$$

\subsection{Inversion of the Radial Distortion Model}

For some applications we need to invert the radial distortion model. For instance, to build the undistorted version of the image it is usually better to use the inverse of the radial distortion model. So we look for a radial function $G(\hat{r})$ such that

$$
\left(\begin{array}{c}
x-x_{c} \\
y-y_{c}
\end{array}\right)=G(\hat{r})\left(\begin{array}{c}
\hat{x}-x_{c} \\
\hat{y}-y_{c}
\end{array}\right)
$$

where

$$
\hat{r}=\sqrt{\left(\hat{x}-x_{c}\right)^{2}+\left(\hat{y}-y_{c}\right)^{2}} .
$$

From the above expression we obtain that

$$
r=G(\hat{r}) \hat{r} .
$$

On the other hand we have

$$
\left(\begin{array}{c}
\hat{x}-x_{c} \\
\hat{y}-y_{c}
\end{array}\right)=L(r)\left(\begin{array}{l}
x-x_{c} \\
y-y_{c}
\end{array}\right),
$$

and therefore

$$
\hat{r}=L(G(\hat{r}) \hat{r}) G(\hat{r}) \hat{r} .
$$


So we conclude that $G(\hat{r})$ is a root of the polynomial

$$
P(\lambda)=1-L(\lambda \hat{r}) \lambda=1-\sum_{j=0}^{N_{k}} k_{j} \hat{r}^{j} \lambda^{j+1} .
$$

In order to minimize the distance between the undistorted point $(\hat{x}, \hat{y})$ we choose, among all possible real roots of $P(\lambda)$, the one nearest to 1 .

\subsection{Lens Distortion Center}

We manage the lens distortion center. Users can, optionally, provide the lens distortion center location and optimize it by minimizing the distance of the collection of primitive points to the associated lines.

\section{Examples}

We present some examples to illustrate the performance of the proposed method.

\subsection{Calibration Pattern}

First, we start by correcting the distortion in a synthetic case, that is, a calibration pattern (Figure 1). The image has been obtained as follows: it has been printed out and we have taken a photo of it. The advantage of the calibration pattern is that we can easily identify the rectangles presented in the image, and automatically select the edge segments and points we will use for the estimation of the distortion model parameters.

The yellow lines displayed on the left image are the lines joining the marked points selected by the user. Note that we know a priori that this yellow line must be straight. The selected points (primitives) for this image are available in a text file as supplementary material ${ }^{3}$. In this file, the number 1 (first line) accounts for the number of lines to be used by the program to correct the distortion (in this case it is only 1, but the number can be arbitrary). The following lines indicates the coordinates (pixels) of the selected points. From the result obtained, it seems that the method works well.

The black areas appearing at the top and at the bottom of the undistorted image are related to the correction procedure and can be removed just by selecting the inside region of interest.

\subsection{Frame}

The second result (Figure 2) is for a color photo showing an important barrel distortion or pincushion distortion. For this case, the user has selected the top border of the frame as a straight reference line. The undistorted image once again reveals that, even when selecting a simple primitive, the distortion is firmly corrected.

\subsection{Building}

The third example (Figure 3) is a color photo of a building (a church). This is an interesting case to appreciate that one can select any a priori known straight line, as it occurs here, where the user has selected two vertical lines, instead of the horizontal lines. The result shows the potential of the method.

\footnotetext{
${ }^{3}$ https://doi.org/10.5201/ipol.2010.ags-alde
} 

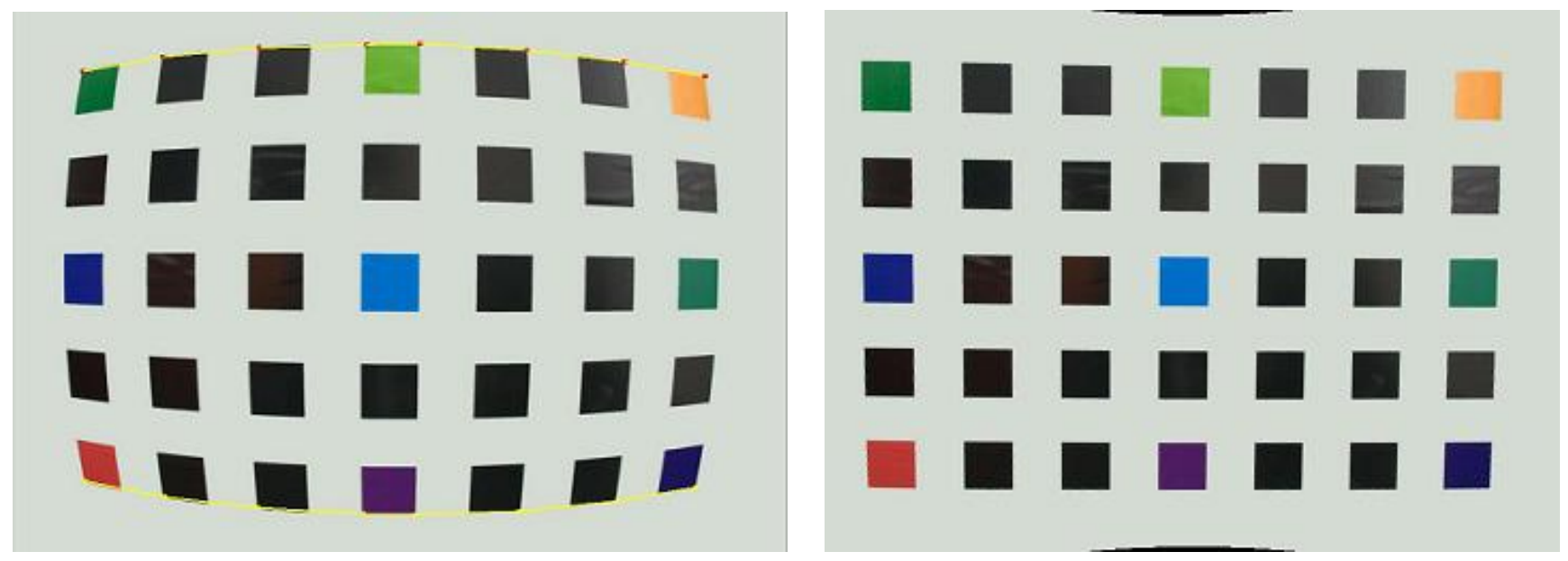

Figure 1: Calibration pattern. Left, distorted original image. Right, corrected image.
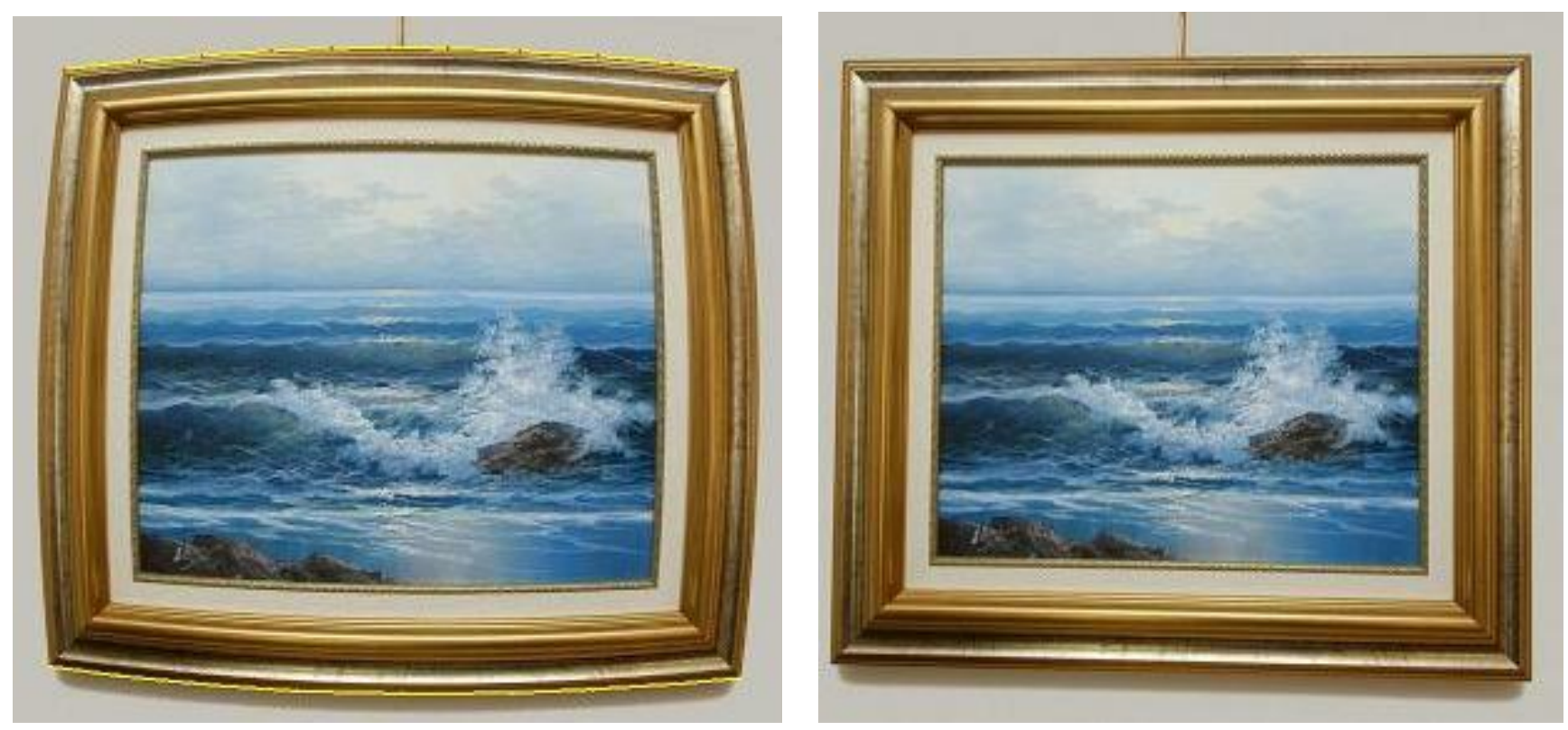

Figure 2: Frame. Left, distorted original image. Right, corrected image.
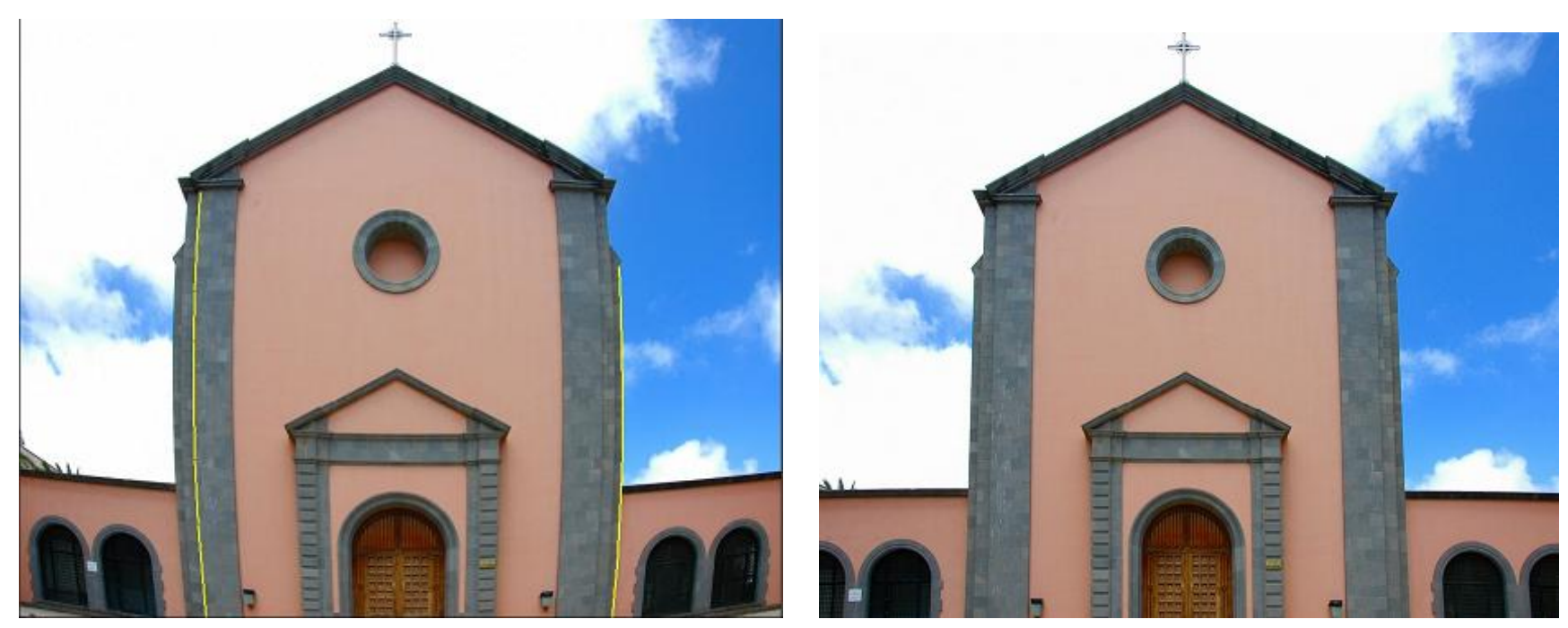

Figure 3: Building. Left, distorted original image. Right, corrected image. 


\section{Image Credits}

All images by the authors (license CC-BY-SA).

\section{References}

[1] Luis Alvarez, Luis Gomez and Rafael Sendra. An Algebraic Approach to Lens Distortion by Line Rectification, Journal of Mathematical Imaging and Vision, vol. 35, n 1, pp. 36 - 50, September 2009. http://dx.doi.org/10.1007/s10851-009-0153-2.

[2] F. Winkler. Polynomial Algorithms in Computer Algebra, Wien New York: Springer-Verlag, 1996. ISBN:3211827595. 